\title{
Colagenosis perforante reactiva adquirida. Caso clínico
}

\author{
CONSUELO CÁRDENAS D. ${ }^{1}$, CRISTINA BELLO C. ${ }^{1}$, PABLO URIBE G. ${ }^{1}$, \\ WILLIAM ROMERO G. ${ }^{1}$, SERGIO GONZÁLEZ B. ${ }^{2}$
}

\section{Acquired reactive perforating collagenosis. Report of two cases}

Acquired reactive perforating collagenosis is a perforating dermatosis characterized by transepidermal elimination of collagen. It is frequently associated to diabetes mellitus and chronic renal insufficiency, but it is also related to other systemic diseases. The lesions tend to resolve once the underlying condition is treated. We report two patients with the condition. A 65 year-old diabetic female on hemodialysis consulted for multiple itching cutaneous ulcers lasting one year. On physical examination, hyperpigmented papules and nodules were observed. A 65 year-old female with chronic renal failure in hemodialysis consulted for itching lesions in hands, forearms and arms. On physical examination, hyperpigmented lesions with ulcers, erosions and crusts were observed. In both cases, the pathological study of the lesions disclosed a reactive perforating collagenosis.

(Rev Med Chile 2010; 138: 1281-1284).

Key words: Collagen diseases; Diabetes mellitus; Skin diseases, vesiculobullous.

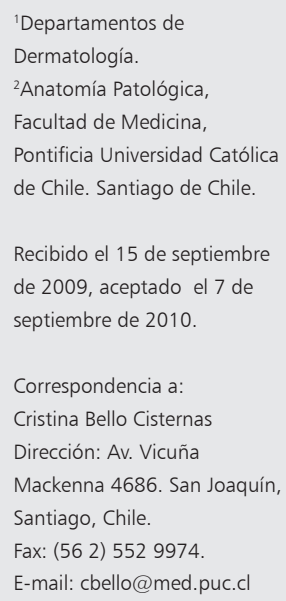

L a colagenosis perforante es una rara enfermedad que pertenece a un grupo heterogéneo de patologías llamado dermatosis perforantes. Este conjunto de enfermedades se caracteriza por la eliminación transepidérmica de varios elementos como queratina, colágeno y fibras elásticas. Tradicionalmente se distinguen cuatro entidades clásicas según el material eliminado y el tipo de disrupción epidérmica que producen: la enfermedad de Kyrle, la elastosis perforante serpiginosa, la foliculitis perforante y la colagenosis perforante reactiva ${ }^{1}$. Estas entidades fueron descritas clásicamente como trastornos genéticos hereditarios de la piel, de aparición generalmente en la infancia ${ }^{2}$. Sin embargo, en las últimas décadas se han descrito varios casos con aparición en la vida adulta en pacientes con diversas enfermedades como la diabetes mellitus y la insuficiencia renal crónica, por lo que se acuñó un nuevo término: dermatosis perforante adquirida. Este término engloba un amplio espectro de desórdenes perforantes de la piel que se presentan en asociación a enfermedades sistémicas ${ }^{3}$.

La colagenosis perforante reactiva es parte del conjunto de enfermedades que conforman las dermatosis perforantes y se caracteriza por la eliminación transepidérmica de bandas alteradas de colágeno ${ }^{4}$. Existen dos formas de presentación de esta enfermedad. La forma clásica es poco frecuente, se hereda como un rasgo autosómico recesivo, se inicia en la infancia sin enfermedades concomitantes y es usualmente asintomática y de resolución espontánea ${ }^{4}$. Por otro lado, la forma adquirida o adulta está frecuentemente asociada a diabetes mellitus y a enfermedades renales, como también a otras enfermedades sistémicas, y se caracteriza por presentar prurito intenso ${ }^{5}$. Clínicamente ambas formas se presentan como múltiples pápulas umbilicadas hiperpigmentadas y nódulos con un tapón central queratósico. Las lesiones se ubican generalmente en las superficies extensoras de las piernas, pero también pueden 
aparecer en los brazos, cabeza y cuello. A menudo se puede observar el fenómeno de Koebner ${ }^{5,6}$, que consiste en la reproducción de las lesiones al realizar una injuria de la piel, y en la tendencia de adoptar la disposición de ésta. Las lesiones tienden a resolverse una vez tratada la patología sistémica subyacente ${ }^{5}$.

Los casos que comunicamos a continuación, muestran la asociación de esta patología cutánea a diabetes mellitus y a insuficiencia renal crónica, las patologías que con mayor frecuencia son asociadas a esta enfermedad. También se reporta la asociación a otras patologías sistémicas como el hipotiroidismo, cardiopatía hipertensiva e hipertensión arterial.

\section{Caso 1}

Paciente de 65 años, sexo femenino, con antecedentes de diabetes mellitus tipo 2 insulinorequirente, insuficiencia renal crónica en hemodiálisis, cardiopatía hipertensiva e hipotiroidismo. Usuaria de carbonato de calcio, fumarato ferroso y levotiroxina. Consultó por úlceras cutáneas múltiples, generalizadas, pruriginosas, de un año de evolución. En el examen físico destacaban pápulas y nódulos hiperpigmentados con centro queratósico (aproximadamente 30), de hasta $1 \mathrm{~cm}$ de diámetro, algunas ulceradas con material blanquecino-grisáceo en el fondo, ubicadas en extremidades, pabellones auriculares y lengua.

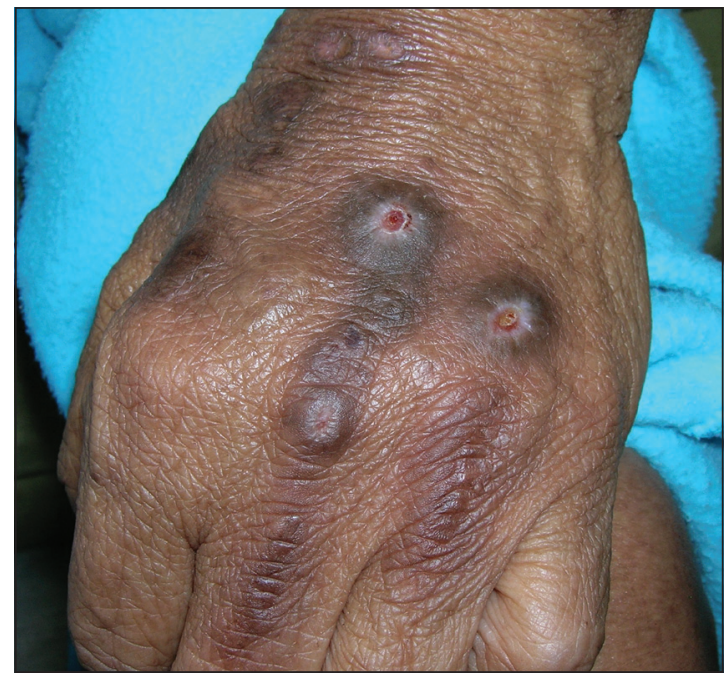

Figura 1. Pápulas hiperqueratósicas ulceradas con material blanquecino-grisáceo en el fondo en dorso de mano.
(Figura 1). Se realizó biopsia de piel, que mostró úlcera con detritus celular y en el fondo fibras elásticas y haces de colágeno de disposición vertical. Epidermis adyacente con hiperqueratosis, acantosis, hipergranulosis y leve pigmentación de queratinocitos basales. Dermis con leve infiltrado inflamatorio linfocitario disperso. Los hallazgos fueron compatibles con colagenosis perforante reactiva. La paciente fue tratada con corticoides tópicos de alta potencia y antihistamínicos orales, con respuesta parcial.

\section{Caso 2}

Paciente de 65 años, sexo femenino, con antecedentes de insuficiencia renal crónica de larga data, trasplante renal en 1999 que rechazó a los

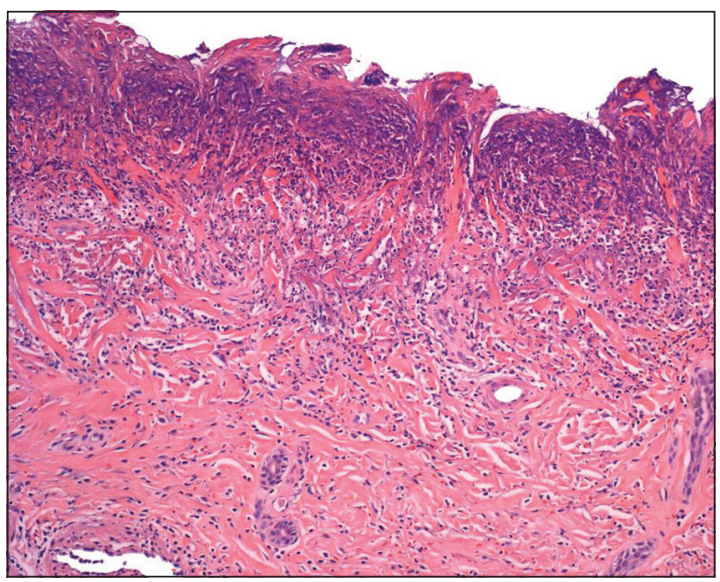

Figura 2. Microfotografía de úlcera con haces de colágeno verticales y detritus celulares. HE, 200x.

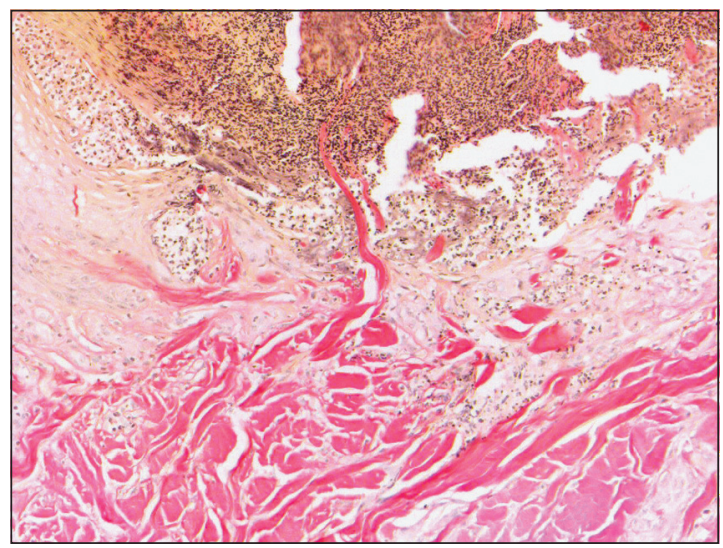

Figura 3. Microfotografía de úlcera con tinción de van Gieson, 200x. 
8 meses, en hemodiálisis trisemanal desde el año 2000 e hipertensión arterial secundaria. Tratada con eritropoyetina, ácido fólico, atenolol y losartan. Consultó por una historia de cuatro meses de evolución, caracterizada por lesiones pruriginosas en manos, antebrazos y espalda. En el examen físico destacaban hiperpigmentación difusa, erosiones, úlceras y pápulas costrosas, ubicadas preferentemente en dorso de manos, antebrazos, tórax anterior y región interescapular. Se realizó biopsia de las lesiones que mostró piel con úlcera y costra fibrinoleucocitaria. El fondo de la úlcera con haces de colágeno de disposición vertical, epidermis adyacente con hiperortoqueratosis, acantosis y leve espongiosis. La dermis con leve infiltrado inflamatorio disperso y perivascular superficial linfocitario, con algunos plasmocitos y neutrófilos y foco hemorrágico. Los hallazgos informados fueron compatibles con colagenosis perforante de tipo reactivo (Figuras 2 y 3 ). Se indicaron antihistamínicos orales y clobetasol 0,05\% + cloranfenicol $2 \%$ con respuesta parcial a un año de seguimiento.

\section{Discusión}

La colagenosis perforante reactiva pertenece a un grupo heterogéneo de patologías llamadas dermatosis perforantes. Fue descrita por primera vez en 1967 por Mehregan et al, como una condición en la cual se elimina colágeno alterado a través de la epidermis ${ }^{4}$. Aunque la forma clásica se describió inicialmente en niños, como un trastorno congénito hereditario, la mayoría de los casos publicados corresponden a la forma adquirida, caracterizada por presentarse en pacientes adultos con patologías sistémicas. Faver et al propusieron ciertos criterios diagnósticos para la forma adquirida de la colagenosis perforante reactiva, estos son: i) hallazgos histológicos de eliminación transepidérmica de bandas de colágeno basófilas necróticas dentro de una depresión epidermal con forma de copa; ii) pápulas umbilicadas o nódulos con un tapón queratósico central adherido; y iii) aparición de las lesiones después de los 18 años de edad $^{7}$. Nuestros pacientes cumplen con los criterios clínicos propuestos por Faver, sin embargo, en el diagnóstico histopatológico no se evidencia necrosis de las bandas de colágeno. Esto coincide con reportes que describen que las fibras colágenas no presentan degeneración obvia ${ }^{8,9}$.
La patogénesis precisa de la colagenosis perforante reactiva adquirida es aún desconocida. Existen varias teorías que explican la patogenia de esta condición. Una de estas teorías involucraría al prurito, síntoma que generalmente se encuentra en las patologías relacionadas a la colagenosis perforante reactiva adquirida. El prurito causaría rascado que provocaría microtrauma. Este microtrauma produciría alteraciones en las fibras colágenas de la dermis papilar provocando su eliminación transepidérmica ${ }^{10}$. Otra teoría propuesta para los casos asociados a la diabetes mellitus, propone que la microangiopatía produciría hipoxia y prurito que dañaría al colágeno ${ }^{11}$, además el mayor estrés oxidativo y la glicosilación produciría entrecruzamiento de éste ${ }^{12}$. En la última década varios estudios han demostrado que altos niveles de fibronectina y otras proteínas de la matriz extracelular, en conjunto con el aumento del TGF- $\beta$ estarían implicados en la patogénesis de la colagenosis perforante reactiva adquirida ${ }^{9,13,14}$.

Las enfermedades que más se asocian a la colagenosis perforante reactiva adquirida son la diabetes mellitus y la insuficiencia renal crónica ${ }^{5-15}$, ambas patologías presentes en nuestras pacientes. En un estudio inglés hecho en una población de insuficientes renales crónicos en hemodiálisis, se demostró que $11 \%$ presentaba colagenosis perforante reactiva adquirida ${ }^{15}$. Además se ha descrito la asociación con otras enfermedades como hipotiroidismo, linfomas, hiperparatiroidismo, neurodermatitis, SIDA, fibrosis pulmonar, enfermedades hepáticas, dermatitis atópica, infección por citomegalovirus, mucormicosis cutánea, síndrome de Treacher Collins, púrpura de Schönlein-Henoch, enfermedad de Mikulicz, reacción adversa a medicamentos, escabiosis y herpes zoster ${ }^{5,16}$. Sin embargo, muchas de estas enfermedades se presentaron asociadas además a diabetes o a falla renal. En muy raras ocasiones la colagenosis perforante reactiva adquirida se ha visto asociada a neoplasias. Se han reportado casos de linfoma, carcinoma hepatocelular, cáncer de próstata, cáncer de colon, carcinoma periampular, metástasis hepáticas de tumor primario desconocido y carcinoma papilar de tiroides ${ }^{17}$. Como aún no se conocen los mecanismos patológicos específicos de la colagenosis perforante reactiva, la relación entre ella y sus enfermedades asociadas aún no es clara.

El diagnóstico diferencial de pápulas o nódulos 
con un tapón queratósico central adherido o con ulceración como se ven en la colagenosis perforante reactiva adquirida puede hacerse clínicamente entre queratoacantomas eruptivos, enfermedad de Darier, desórdenes foliculares como la queratosis pilar, eritema indurado, sarcoidosis, infecciones fúngicas profundas, sarcoma de Kaposi, pitiriasis liquenoide y varioliforme aguda y frinoderma ${ }^{17,18}$. En el contexto de la insuficiencia renal crónica el diagnóstico diferencial debe incluir patologías relacionadas al prurito urémico como otras dermatosis perforantes adquirida, liquen plano, prurigo nodularis, porfiria, pseudoporfiria, calcifilaxis, $y$ dermatosis bulosa por $\operatorname{IgA}^{19}$.

El tratamiento de la colagenosis perforante no es siempre satisfactorio. Se han intentado múltiples opciones terapéuticas, entre ellas corticoides tópicos, retinoides, queratolíticos, antihistamínicos, crioterapia con nitrógeno líquido, fototerapia, y alopurinol, todas con distintos grados de éxito ${ }^{20}$. Sin embargo, el manejo del prurito y el control de las enfermedades de base se han considerado como los pilares fundamentales de la terapia.

\section{Referencias}

1. Patterson JW. The perforating disorders. J Am Acad Dermatol 1984; 10: 561-81.

2. Patterson JW. Progress in the perforating dermatoses. Arch Dermatol 1989; 125: 1121-3.

3. Rapini RP, Herbert AA, Drucker CR. Acquired perforating dermatosis: evidence for combined transepidermal elimination of both collagen and elastic fibers. Arch Dermatol 1989; 125: 1074-8.

4. Mehregan AH, Schwartz OD, Livingood CS. Reactive perforating collagenosis. Arch Dermatol 1967; 96: 277 82.

5. Yadav MK, Sangal BC, Bhargav P, Jai PR, Goyal M. Reactive perforating collagenosis. Indian J Pathol Microbiol 2009; 52: 106-7.

6. Hoque SR, Ameen M, Holden CA. Acquired reactive perforating collagenosis: four patients with a giant variant treated with allopurinol. Br J Dermatol 2006; 154: 759-62.

7. Faver IR, Daoud MS, Su WPD. Acquired perforating collagenosis. Report of six cases and review of the lite- rature. J Am Acad Dermatol 1994; 30: 575-80.

8. Yanagihara M, Fujita T, Shirasaki A, Ishiguro K, Kawahara K, Ueda K. The pathogenesis of the transepithelial elimination of the collagen bundles in acquired reactive perforating collagenosis. A light and electron microscopical study. J Cutan Pathol 1996; 23: 398-403.

9. Morgan MB, Truitt CA, Taira J, Somach S, Pitha JV, Everett MA. Fibronectin and the extracellular matrix in the perforating disorders of the skin. Am J Dermatopatholol 1998; 20: 147-54.

10. Thiele-Ochel S, Schneider LA, Reinhold K, Hunzelmann N, Krieg T, Scharffetter-Kochanek K. Acquired perforating collagenosis: is it due to damage by scratching? $\mathrm{Br} \mathrm{J}$ Dermatol 2001; 145: 173-4.

11. Kawakami T, Saito R. Acquired reactive perforating collagenosis associated with diabetes mellitus: eight cases that meet Faver's criteria. Br J Dermatol 1999; 140: 5214.

12. Munch M, Balsev E, Jemec GBE. Treatment of perforating collagenosis of diabetes and renal failure with allopurinol. Clin Exp Dermatol 2000; 25: 615-6.

13. Tsuboi H, Katsuoka K. Characteristics of acquired reactive perforating collagenosis. J Dermatol 2007; 34: 640-4.

14. Gambichler T, Birkner L, Stücker M, Othlinghaus N, Altmeyer P, Kreuter A. Up-regulation of transforming growth factor-beta3 and extracellular matrix proteins in acquired reactive perforating collagenosis. J Am Acad Dermatol 2009; 60: 463-9.

15. Morton CA, Henderson IS, Jones MC, Lowe JG. Acquired perforating dermatosis in a British dialysis population. Br J Dermatol 1996; 135: 671-7.

16. Shiomi T, Yoshida Y, Horie Y, Yamamoto O. Acquired reactive perforating collagenosis with the histological features of IgG4-related sclerosing disease in a patient with Mikulicz's disease. Pathol Int 2009; 59:326-31.

17. Yazdi S, Saadat P, Young S, Hamidi R, Vadmal MS. Acquired reactive perforating collagenosis associated with papillary thyroid carcinoma: a paraneoplastic phenomenon? Clin Exp Dermatol 2010; 35: 152-5.

18. Basak P, Turkmen C. Acquired reactive perforating collagenosis. Eur J Dermatol 2001; 11: 466-8.

19. Kurban Ms, Boueiz A, Kibbi AG. Cutaneous manifestations of chronic kidney disease. Clin Dermatol 2008; 26: 255-64.

20. Ghosh Sk, Bandyopadhyay D, Chatterjee G. Acquired reactive perforating collagenosis following insect bite. Indian J Dermatol Venereol Leprol 2009; 75: 306-7. 